\title{
Investing in brain-based memory leads to decreased use of technology-
}

\section{based memory}

\author{
Patrick P. Weis \& Eva Wiese \\ George Mason University, USA
}

\begin{abstract}
Author's Notes:
Author contributions. PPW and EW conceived and designed the study. Data collection was performed under supervision of PPW. PPW performed the data analysis and interpreted the data together with EW. PPW and EW wrote the manuscript. PPW and EW approved the final version of the manuscript for submission.

Acknowledgements. We thank our undergraduate RA Caitlin Mahoney for collecting the data. All data and the R analysis script are available at https://osf.io/scbj3/.
\end{abstract}

\section{Corresponding author:}

Patrick P. Weis

Email: pweis@gmu.edu

(C) 2019. This manuscript version is made available under the CC-BY-NC-ND 4.0 license http://creativecommons.org/licenses/by-nc-nd/4.0/ .

The final version of this manuscript has been published in Journal of Experimental Psychology: Applied at https://doi.org/10.1037/xap0000259 . 


\section{Abstract}

Humans frequently use external (environment-based) strategies to supplement their internal (brain-based) thought. In the memory domain, whether to solve a problem using external or internal retrieval depends on the accessibility of external information, judgment of mnemonic ability, and on the problem's visual features. It likely also depends on the accessibility of internal information. Here, we asked whether internal accessibility contributes to strategy choice even when visual features bear no information on internal accessibility. Specifically, 114 participants were to validate alphanumerical equations (e.g., $\mathrm{A}+2=\mathrm{C}$ ) whose visual appearance (addends 2, 3, or 4) signified different difficulty levels. First, some equations were presented more frequently than others, allowing participants to establish efficient internal access to the correct solution via memory retrieval rather than counting up the alphabet. Second, participants viewed the equations again but could access the correct solution externally using a computer mouse. We hypothesized that external strategy use should selectively decrease for frequently learned equations and irrespectively of the task's visual features. Results mostly confirm our hypothesis. Exploratory analyses further suggest that participants partially used a sequential "try-internal-retrieval-first" mechanism to establish the adaptive behavior. Implications for intervention methods aimed at improving interactive cognition are discussed.

\section{Public Significance Statement}

In the present study, human problem solvers were less inclined to retrieve information stored externally on a computer when they had the chance to save that information in internal brainbased memory. The results suggest that (1) humans have the skills to proficiently incorporate external information storages into their thought processes and (2) that improving brain-based 
memory automatically decreases reliance on external information storages like computers or smartphones.

\section{Keywords}

Cognitive Offloading; Strategy Selection; Distributed Cognition; Problem Solving; Information Access Cost 


\section{Introduction}

Imagine you are in your kitchen and about to prepare your new favorite meal that got a fivestar rating on your go-to recipe website. You prepared it once and are known for your good memory. The next time you prepare the same dish, would you try to find the recipe online again or would you rely on your mnemonic abilities? Human problem solvers face similar problems, i.e. whether to retrieve information from internal (brain-based) or external (environment-based; e.g., internet, paper) storage, on a daily basis. The present study is designed to illuminate the underlying decision process. Its focus is on investigating the impact of internal information accessibility (i.e., performance of memory retrieval) on the use frequency of external information storages.

\section{The Paradigm: Solving Alphanumerical Equations with External Storage}

To facilitate the understanding of the remaining introduction, we now briefly describe the paradigm we designed for the present inquiry before continuing with theoretical considerations. Each trial, participants were faced with alphanumerical problems of the format "Letter + Number $=$ Letter" and asked to indicate whether counting the indicated number up the alphabet from the former letter equals in the latter letter. In a mixed design, we altered the number (factor Addend: 2, 3, or 4) within participants and the frequency with which participants learned solving specific problems involving the different Addends (factor Learning: 2 or 4$)^{1}$ between participants. After solving specific alphanumeric problems frequently (here, 128 times), human problem solvers are known to shift away from a slow counting to a fast memory retrieval strategy (Compton \& Logan, 1991). This strategy shift prominently alters

\footnotetext{
${ }^{1}$ Learning 2: 128 trials with the " 2 " Addend, 64 trials with the " 3 " Addend, and 32 trials with the " 4 " Addend; Learning 4: 32 trials with the " 2 " Addend, 64 trials with the " 3 " Addend, and 128 trials with the " 4 " Addend
} 
the information's internal accessibility. After the accessibility of internal information had been altered, participants gained access to an external storage that could be used to replace internal cognitive strategies. Specifically, participants gained access to a black box that revealed the correct solution whenever the mouse cursor was being moved on top of it (e.g., it would reveal " $\mathrm{D}$ " if the verification task was " $\mathrm{A}+2=\mathrm{C}$ "). This design allowed analyzing whether participants accessed the black box less frequently for equations with high internal accessibility. More generally, it afforded insight into how proficiently human problem solvers incorporate technology-infused environments into their memory processing.

\section{Interactive Cognition: Distributed, Embodied, and Situated Perspectives on Cognitive Sci- ence}

Researching human cognition using interactive paradigms like the one just described has been a focus in recent cognitive science research. The subfields of distributed (e.g., Hollan, Hutchins, \& Kirsh, 2000), embodied (e.g., Clark, 1999; Wilson, 2002), and situated (e.g., Robbins \& Aydede, 2009) cognition are focused on researching the cross-play between sensory input, brain-based cognition, motor output, and manipulation of the environment unfolding over time. One upshot of such a holistic ${ }^{2}$ perspective on cognition is that it enables researching cognition-related behaviors in real-world complex environments like today's and tomorrow's technologized homes and workplaces. Relating back to the cooking example, this means that a recipe does not need to be retrieved from brain-based memory (knowledge in the

\footnotetext{
${ }^{2}$ Traditionally, cognitive science research has been based on the less holistic sequential information processing model, focusing on how brain-based cognition transforms peripheral input into muscular output. In that tradition, cognition is defined as the central element in a sequential process - from input, to central processing, to output (also called the solipsistic perspective).
} 
head; Norman, 1988) but could instead be retrieved from environment-based sources like paper notes or the internet (knowledge in the world; Norman, 1988; for a review comparing brain-based and internet-based information retrieval, see Clowes, 2013). Without knowledge in the world, the only option to access information would be to use brain-based memory. With knowledge in the world however, the cost structure of the human inferential landscape (Kirsh, 2010) changes. The world can then provide problem solvers with knowledge similar to the one available in the brain but associated with different retrieval costs. Thus, to understand cognitive operations like information retrieval in situations in which the environment can be exploited, it is imperative to broaden the focus beyond the brain. To illustrate this issue, imagine the following scenario that is focused on understanding Peter's cognitive operations: you observed Peter preparing meals throughout the whole last week. You noticed that he is hardly ever looking up recipes on his smartphone. Why would that be?

\section{Accessibility of External Information Influences External Information Retrieval}

Proficient problem solvers need to refrain from integrating external information when it is associated with higher costs than relying on internal strategies. Thus, possibly, Peter is experiencing poor network coverage and loading the recipe page would take too long to be beneficial. Current literature suggests that there is some truth to this option: human problem solvers proficiently adjust the frequency of external information retrieval based on the accessibility of the external information.

In previous studies, accessibility of external information has been altered via a delay between the time when externally stored information was requested and when it eventually showed up (Gray, Sims, Fu, \& Schoelles, 2006; Morgan, Patrick, Waldron, King, \& Patrick, 2009; Walsh \& Anderson, 2009), by altering the size of the interface elements needed to access the information (which manipulates time costs in a more natural manner via Fitts' law; 
Gray et al., 2006), by altering the number of key strokes needed to change what external information is shown (O'Hara \& Payne, 1998), or by altering the distance between the problem solver and a computer from which the relevant information could be accessed from (Storm, Stone, \& Benjamin, 2017). In all cases, decreased accessibility led to less external information retrieval and, equivalently, to more internal information retrieval. In one study, an ideal performer analysis suggested that problem solvers switched between using internal and external information retrieval in a way that maximizes speed (Gray et al., 2006); in another study, problem solvers were found to proficiently switch between internal and external strategies as to maximize a monetary performance-related reward (though there was a slight bias for internal strategies and, strictly speaking, participants were found to probability match rather than optimize; Walsh \& Anderson, 2009).

Taken together, the studies suggest that problem solvers adjust their use of external information based on properties of the external information source (i.e., accessibility). Changing the accessibility of externally stored information can alter the cost structure of the inferential landscape which causes agents equipped with a rational and adaptive cognitive system (which might be close to the human cognitive system; Anderson, 1990) to adjust how they incorporate the environment into their cognitive processing.

\section{Additional Factors Influencing External Information Retrieval}

If human problem solvers are indeed able to mix internal and external cognitive strategies to maximize performance, they should not only be sensitive to properties of external information sources. Instead, they should pay equal attention to features of the task (e.g., does the task look difficult?) and properties of internal brain-based information sources (e.g., how fast is it to access the solution internally?). For example, after using a written recipe for preparing a meal for more than ten times, a substantial brain-based memory trace of the recipe 
should have built up that might make it more efficient for a culinary problem solver to omit using the written recipe and rely on internal information instead.

And indeed, humans can be quite proficient in deciding between internal and external strategies even when the cost of external information access is kept constant (e.g.; Risko, Medimorec, Chisholm, \& Kingstone, 2014; Siegler \& Lemaire, 1997; Walsh \& Anderson, 2009). When allowed to use internal (e.g., internal counting) and external (i.e., using a calculator) strategies for arithmetic problem solving, participants were repeatedly shown to mix the strategies in a way that led to better speed performance than when allowed to only use one of the strategies (e.g., Siegler \& Lemaire, 1997; Walsh \& Anderson, 2009). More specifically, participants quite adaptively preferred using mental arithmetic for equations in which one multiplicand was "10" (e.g., $17 \times 10$ ) and the calculator for tasks in which no multiplicand was “10” (e.g., 17 x 13; Siegler \& Lemaire, 1997).

But how is such adaptive external strategy use being achieved and what is the driving force behind adaptive cognitive strategy selection? In the following, we are discussing four possible candidates: 1. feature-specific strategy selection (e.g., seeing a "10" as a factor in an arithmetic task affords the specific internal strategy of simply adding a 0 to the other factor to get the product), 2. feature-based apparent difficulty of the problem (e.g., seeing a "10" as a factor is associated with an easy problem through metacognitive reasoning), 3. evaluation of internal accessibility of the solution (e.g., perceiving oneself as being bad at math and thus preferring external over internal strategies), and 4. actual internal accessibility of the solution (e.g., the solution to " $17 \times 10$ " is quick to calculate internally).

1. Thoroughly analyzing the visual features of a problem is likely a highly relevant process for adaptive cognitive strategy selection. Feature-specific strategy selection has been frequently researched by observing arithmetic problem solvers. For 
example, simply seeing a "10" had profound consequences for problem solvers" strategy selection: it accounted for a 14 percentage point increase in explained strategy choice variance on top of the variance explained by reaction time differences between internal and external strategies (Siegler \& Lemaire, 1997; Experiment 1). A similar effect of visual features on strategy choice has been triggered by a "5" as a factor in arithmetic-based problems (Lemaire \& Reder, 1999; Experiment 3$)^{3}$ or by a letter that enabled rule-based instead of retrieval-based processing in a string classification task (e.g., if the first letter of the string is a consonant, classify the string as "code" instead of "noncode"; Bourne, Raymond, \& Healy, 2010).

2. A second relevant process requiring analysis of a problem's visual features constitutes metacognitive judgments related to the problem's difficulty. For example, it has been suggested that some problem solvers assume that it is manageable to keep an array of ten letters in working memory, which is why they skip writing the letters down and end up with attenuated task performance (Risko \& Dunn, 2015). Similarly, it has been suggested that whether adopting an external or an internal strategy heavily depends on the familiarity of the respective problem rather than how well the solution can be accessed internally (Schunn, Reder, \& Nhouyvanisvong, 1997). Note that familiarity-based judgments might not be made on a

\footnotetext{
${ }^{3}$ However, note that participants do not always use that rule but, adaptively, skip the rule in experimental sessions in which the last digit hardly violates the five rule (Lemaire \& Reder, 1999; Experiment 3). Human problem solvers thus also exhibit feature-independent strategy adaptation.
} 
conscious level but depend on an implicit frequency tracker (Onyper, Hoyer, \& Cerella, 2006).

3. The third process relevant for cognitive strategy choice is the evaluation of own performance. Such evaluation can have components independent of actual skill (Gilbert, 2015) and might be a reason for memory avoidance in older adults (Hines, Hertzog, \& Touron, 2012; Touron, 2015).

4. A fourth relevant process might be the actual - in contrast to the estimated - internal accessibility of the solution: how fast can one produce a correct solution with mental processing? Although adaptive selection between an internal and an external strategy has been frequently shown, it is hard to say whether the problem solver's sensitivity to brain-based task performance actually contributed to strategy selection. Instead, as pointed out in the above, problem solvers might rely on what can be directly seen. For example, differences in task difficulty are usually obvious (e.g., 17 x 13 looks harder than 7 x 10) and properties of external information sources are oftentimes equally observable (e.g., accessing information using a slow internet connection makes you stare at a mostly empty screen for a while). In comparison, perceiving the properties of internal information sources might prove challenging. As mentioned in the preceding paragraph, judgments of task-related internal memory ability can be independent of actual ability (Gilbert, 2015; Experiment 1). Given the difficulty of accurately judging own ability and the frequent reliance on visible task features for deciding between internal and external cognitive processing, it seems questionable how big the influence of internal memory accessibility on proficient external strategy use really is. 


\section{Influence of Accessibility of Internal Information on External Information Retrieval}

So far, we have argued the accessibility of external information, judgments of own skill, and visual features of the task influence how problem solvers mix internal and external strategies. We have also argued that, based on the current evidence, it is questionable whether the same holds for the accessibility of internal information. Consequently, with the current study, we aim to investigate whether problem solvers monitor their internal information access to inform the orchestration process between internal and external information retrieval or whether they prefer possibly misleading external cues and higher-level metacognitive evaluations (like evaluations of own skill) for the same end. However, drawing on the previous studies on cognitive strategy selection and cognitive offloading that are discussed in what follows, we hypothesize that human problem solvers can indeed adapt their interactive behavior based on internal information accessibility and independent of solely feature-based reasoning.

In one study (Howes, Duggan, Kalidindi, Tseng, \& Lewis, 2016), problem solvers were tested on their ability to copy name lists. Initially, in a no-choice condition, problem solvers had to copy lists consisting of between three and nine names. Then, in a choice condition, people were able to select their preferred list length on their own. Results show that problem solvers tended to choose the list length they performed best with in the no-choice condition. Promisingly, the study shows that problem solvers are able to adapt their interaction behavior depending on how well information can be stored in internal working memory. Importantly however, the study allowed participants only to alter the set size parameter of an internal process (i.e., storing words in working memory). Though while the results sound 
promising, the study did not investigate how participants chose between internal and external strategies, which is the focus of the current investigation ${ }^{4}$.

In another study (Touron \& Hertzog, 2004), problem solvers had to decide whether one noun was "correctly" paired with another noun. To find out whether a noun was paired correctly, problem solvers could rely on two strategies. At the beginning, they only could use an external strategy: they could search through a list of noun pairs provided on screen and compare whether the nouns that were paired in the current problem were also paired in the list on screen. After a while, problem solvers could also use an internal strategy: if they already had had the opportunity to learn parts of the list in earlier trials, they could consult their memory. Results showed that problem solvers relied less on the external search strategy once they had established a good enough memory to use the internal strategy. The only limiting factor of this study is that internal strategies had become available through learning while external strategies were available from the very beginning. Thus, it was to be expected that problem solvers used external information for unlearned problems since no internal options were available. Other paradigms like arithmetic (Walsh \& Anderson, 2009) or alphanumeric (Compton \& Logan, 1991; Zbrodoff, 1995) problem solving avoid this lack of an internal option by providing an internal strategy that is computation- instead of retrieval-based. In the current study, we decided to use an alphanumeric task to avoid the unavailability of an internal strategy.

Some correlational evidence also suggests that problem solvers adjust how frequently they accessed external information based on internal information accessibility. For example,

\footnotetext{
${ }^{4}$ A similar finding that suggests adaptive interaction behavior, though also not allowing participants to select between different interaction strategies, was reported by Neth and Payne (2001).
} 
the older elderly people get, the more they use external information, which is likely an adjustment made due to declining internal information accessibility (Dixon \& de Frias, 2004; Touron, 2015) $)^{5}$. Similar adjustments are compatible with the results of a study examining prospective memory: the better participants performed without the opportunity to use external information, the less they made use of external information when gaining the opportunity to do so (Gilbert, 2015). Further support for the importance of the accessibility of internal information comes from a study comparing two different internal computational strategies (which, in the terminology of this paper, would be equal to two different internal information sources with differential accessibilities) to convert currencies: after briefly practicing the two competing strategies, participants were more likely to use the faster one (Lemaire \& Lecacheur, 2001). Thus, problem solvers can adapt to the accessibility of internal information when selecting between internal strategies. However, note that selecting between internal and external strategies involves additional mechanisms like metacognitive evaluation of the external resource (reviewed by Risko \& Gilbert, 2016) and evaluation of observable performance cues (e.g., size of a button or delay after pressing a button; Gray et al., 2006).

\section{Current Study}

Humans are known to be proficient problem solvers who make use of a variety of internal cognitive strategies to meet their goals (Compton \& Logan, 1991; Lemaire \& Lecacheur, 2001; Lemaire \& Reder, 1999). Evidence is accumulating that humans are also proficient in incorporating external strategies when solving problems (e.g., using a calculator instead of internal strategies; Siegler \& Lemaire, 1997; Walsh \& Anderson, 2009). However,

\footnotetext{
${ }^{5}$ Please note, however, that elderly adults likely over-use external information due to low confidence in their internal memory (reviewed in Touron, 2015).
} 
the importance of feature-based considerations for the selection between internal and external strategies makes it hard to gauge how important sensitivity to the performance of internal strategies for this selection process is (see section: What else Influences External Information Retrieval?).

In the present study, we manipulated the performance of internal problem solving strategies and observed whether participants adjust their use of an external problem solving strategy accordingly. Importantly, we were controlling for the visual features of the task. In other words, we used a paradigm in which an identical problem will be easy to solve internally for one group of participants but hard to solve internally for another group of participants. Based on the studies presented in the preceding section (see section: Does Accessibility of Internal Information Influence External Information Retrieval as well?), we assume changes in the efficiency of the internal strategies to drive changes in how frequently the external strategy is being employed. We expect such adaptive changes in external strategy use despite the fact that participants cannot rely on feature-based reasoning to make adaptive strategy choices. Such a finding would confirm the human problem solver's proficiency in using the environment for cognitive processing.

The present paradigm combines three features that are of particular importance for the interpretability of the study's outcome: (1) The cognitive process investigated here is information retrieval. Thus, external validity is likely highest in the memory domain. (2) In many paradigms, internal accessibility can be derived from appearance. Here, internal information accessibility is manipulated independently from apparent task difficulty. (3) The external strategy used in the current paradigm constitutes using the mouse to access task-relevant information. Thus, external validity is likely highest in the human-computer-interaction domain. 


\section{Methods}

\section{Participants}

In total, 114 undergraduate students participated in the experiment. Two participants were excluded because they reported that they did not understand the task, one because of technical problems, and twelve due to poor task performance (i.e. answering incorrectly in more than $15 \%$ of the problems), resulting in a final sample size of 99 participants (62 females; mean age: 20.3 ; range: $18-50 ; 79$ right handed). 51 participants ( 31 females; mean age: 21.2 ; range: $18-50 ; 37$ right handed) were assigned to the Learning 2 and 48 participants (31 females; mean age: 19.3; range: 18 - 27; 42 right handed) to the Learning 4 condition (see Design and Procedure for details on the Learning factor). Our targeted sample size ( $\mathrm{N}$ of 100) was based on an a-priori power analysis for a within-between interaction at medium effect size conducted in G*Power (Faul, Erdfelder, Lang, \& Buchner, 2007). All participants were recruited from the psychology undergraduate student pool at George Mason University and reimbursed via research participation credits. All participants were at least 18 years old and reported normal or corrected to normal vision. The Ethics Committee at George Mason University approved the experiment and participants provided informed consent prior to participation.

\section{Apparatus}

The experiment was presented at a distance of about $57 \mathrm{~cm}$ on an ASUS VB198T-P 19-inch monitor set to a resolution of $1280 \times 1024$ pixels and a refresh rate of $60 \mathrm{~Hz}$ using MATLAB version R2015b (The Mathworks, Inc., Natick, MA, United States) and the Psychophysics Toolbox (Brainard, 1997; Pelli, 1997). Responses were recorded using a USBconnected standard keyboard and a USB-connected optical mouse with a resolution of 800 
dpi. The mouse cursor speed was set in a way that moving the mouse for $1 \mathrm{~cm}$ would move the cursor on screen for $1.4 \mathrm{~cm}$.

\section{Stimuli}

Stimuli consisted of equations that started with one of six letters (A to F) and had one of three addends ( 2 to 4 ), e.g. " $\mathrm{A}+2=\mathrm{C}$ ". Each equation was presented with a correct or an incorrect solution. The incorrect solution was always one letter further up the alphabet than the correct solution. Thus, in total, 36 different equations were used (18 correct, 18 incorrect). For each participant, each starting letter was uniquely associated with one addend. Associations between starting letters and addends were balanced between participants within each condition in a way that each addend was associated with each target letter with equal probability. Equations were presented on the left side of the screen with an eccentricity of $7^{\circ}$ visual angle. Equations had a width of $5^{\circ}$ and a height of $0.6^{\circ}$. During the second block, a square target box was presented at the right side of the screen with an eccentricity of $7^{\circ}$ and a width of $0.8^{\circ}$.

Task

During the main experiment, participants had to examine the correctness of alphanumerical equations (e.g., A $+2=\mathrm{C}$; see Stimuli) as used by Compton and Logan (1991) to study the transition from solving a task algorithmically to solving it through memory retrieval. Participants had to press the downward arrow key labeled with a checkmark to indicate a correct equation and the upward arrow key labeled with a cross to indicate an incorrect equation. During different parts of the experiment, participants had different options to arrive at their answer. 
During the first part of the experiment, the learning block, participants had the two options investigated by Compton and Logan (1991). First, participants could count upwards the alphabet starting from the first letter given in the equation (internal counting strategy; e.g., when given the equation " $\mathrm{C}+3=\mathrm{G}$ ", counting $\mathrm{C}+1=\mathrm{D}, \mathrm{D}+1=\mathrm{E}$, and $\mathrm{E}+1=\mathrm{F}$, would lead to the conclusion that $\mathrm{C}+3 !=\mathrm{G})$. Second, with increasing exposure to a specific equation, the counting strategy could be replaced by a more efficient memory-based strategy, i.e. participants could recall the solution from memory (internal retrieval strategy). The likely reason for the strategy switch over time is that with increased exposure, the memory trace linking cue (here, the left side of the equation, e.g. $\mathrm{C}+3$ ) and the correct solution (i.e.., F) grows stronger, leading to both increased frequency and increased speed of internal memory recall (as argued by Compton \& Logan, 1991). During the second part of the experiment, the choice block, participants additionally were able to access the solution by hovering the mouse cursor over a black box that then would disappear and reveal the correct solution (external strategy). If an incorrect answer was given or an unassigned key pressed, a feedback message was displayed for $500 \mathrm{~ms}$ immediately after their response. To keep timing constant, the inter-trial interval was shortened to $1500 \mathrm{~ms}$ after incorrect answers. The task is illustrated in Figure 1.

For a more detailed treatise on how problem solvers could automate cue-specific information retrieval, the interested reader can consult Logan's (1988) Instance Theory of Automatization. For a more general overview of how humans create internal problem solving routines, interested readers should consult Anderson's (1987) review article about skill acquisition. For the purpose of the current study, it should be sufficient to know that our problem solvers can choose between an automatic internal retrieval strategy (as in Logan, 1988) and two algorithmic strategies (one internal, one external) to solve a problem and that increased 
exposure with a specific alphanumeric equation increases the likelihood that its solution can be automatically retrieved from internal memory (Logan \& Klapp, 1991).

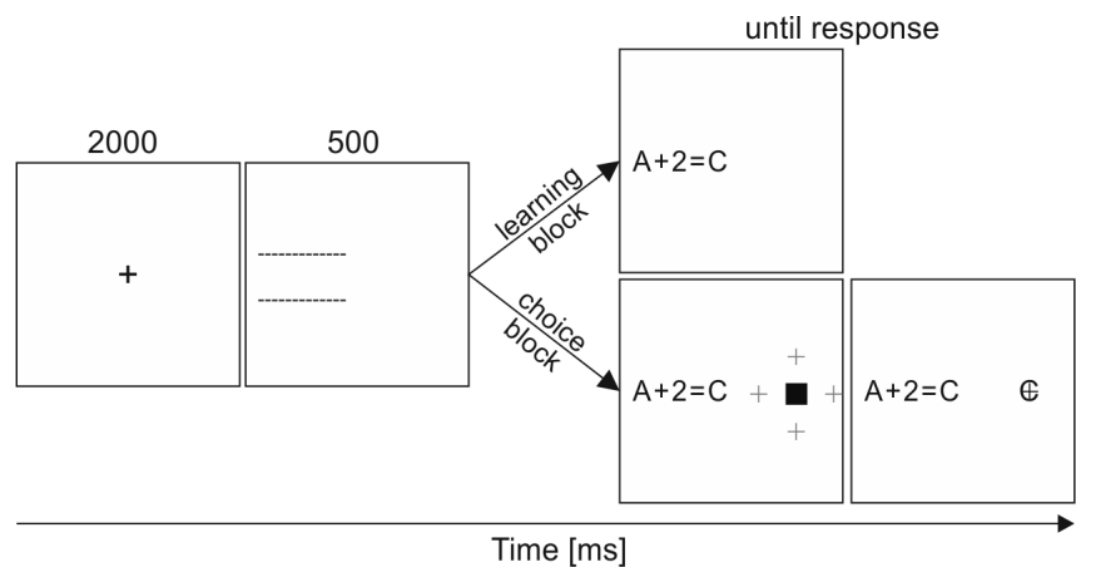

Figure 1. Trial Sequence: Participants have to check alphanumerical equations for correctness via button press (see Task for details). At the beginning of a trial, participants fixate on a fixation cross for $2000 \mathrm{~ms}$ (inter-trial interval). Afterward, to guide the participant's spatial attention, the location of the upcoming equation is indicated for $500 \mathrm{~ms}$. In the learning block, participants have to rely on brain-based internal strategies to solve the equation. In the choice block, participants can additionally rely on an external strategy: hovering with the cursor over the black box will reveal the correct answer hidden under the box (here: "C"). At the beginning of each choice trial, the cursor will appear randomly at one of the four locations indicated by the gray cursors.

\section{Design and Procedure}

The main task followed a $3 \times 2$ mixed design with the within-participants factor Addend (2, 3, or 4) and the between-participants factor Learning ( 2 or 4$)$. The Addend manipulation refers to the addend used in the respective equation (e.g., "2" in the equation $\mathrm{A}+2=\mathrm{C}$ ). The Learning manipulation refers to the addend-specific learning process that took place during the learning block: participants either solved 128 equations with the "2" Addend, 64 equations with the " 3 " Addend, and 32 equations with the "4" Addend (Learning 2) or 32 equations with the "2" Addend, 64 equations with the "3" Addend, and 128 equations with the "4" Addend (Learning 4). This differential learning is known to alter internal information accessibility because problem solvers transition from the time-consuming internal counting strate- 
gy to the more efficient but learning-dependent internal retrieval strategy (compare Figure 3 in Compton \& Logan, 1991). It is important to note that this design affords researching the influence of internal information accessibility on external information retrieval independently of appearance-related effects (e.g., "A + 4" looks inefficient to solve by using internal strategies but is actually very efficient to solve internally for problem solvers in the Learning 4 condition). Also note that we have not altered the internal accessibility of solutions to trials with the " 3 " Addend. Equations with the " 3 " Addend were presented at medium frequency (i.e., 64 trials) for all participants to serve as a baseline or control condition and to make results more comparable to Compton and Logan (1991).

In total, participants engaged in 224 learning trials (128 trials with the well-learned Addend, 64 control trials with the Addend " 3 ", and 32 trials with the remaining Addend) in block one and 96 choice trials (32 with each Addend) in block two. Trial order was randomized. At the beginning of each block, participants additionally engaged in four practice trials (two with addend 1, two with addend 5) with letters different from the ones used in the main task.

Upon entering the lab, participants were welcomed, seated in front of a computer screen, and provided informed consent. During each session, up to three participants were tested simultaneously. Participants then engaged in solving alphanumerical equations during the learning and the choice block, took a brief demographic survey, and finally took a brief metacognitive survey. Overall, the experiment took about 30 minutes to complete. Participants were instructed to respond as quickly and accurately as possible. We refrained from asking participants to exclusively rely on either accuracy or speed because focusing on speed bares the risk of fast but random answers (i.e., participants make use of neither internal nor external strategies) while focusing on accuracy bares the risk of time-intensive double- 
checking (i.e., participants make use of both internal and external strategies for the same problem). Also, since the Learning manipulation likely increases both speed and accuracy for internally solving well-learned problems (compare Compton \& Logan, 1991), asking participants to consider speed and accuracy likely maximizes our effect size.

To keep body posture constant between blocks, participants were asked to respond with their left hand only during the learning block. During the choice block, participants were asked to respond with their left hand and use their right hand to move the mouse when needed.

Analysis

All trials with extreme reaction times above $15 \mathrm{~s}(0.1 \%$ of all trials $)$ were excluded from analysis. After removing these trials, all trials that deviated more than three standard deviations from the individual reaction time means of the respective problem size condition in the respective block ( $1.7 \%$ of all trials) were excluded as well because they likely presented either motor slips (low RTs) or inattentiveness (high RTs). To increase readability, statistical analyses are described directly preceding the respective result. All $p$-values are reported Greenhouse-Geisser-corrected where indicated. 


\section{Results}

Does learning alter how efficiently a solution can be accessed internally?

This analysis served as a manipulation check. The main experiment was divided into two blocks, the learning and the choice block. The purpose of the learning block was to manipulate the ease of internal access to the solutions of the alphanumerical problems (Learning manipulation). Participants in the Learning 2 condition are thus expected to get more efficient in solving equations with the Addend 2 whereas participants in the Learning 4 condition are expected to get more efficient in solving equations with the Addend 4 throughout the learning block. Efficiency in solving equations with the Addend 3 should be comparable across both Learning conditions since learning was kept constant. Meeting these expectations is a crucial prerequisite for the validity of our main analysis (see next section).

To check whether the expectations are met, a $2 \times 3 \times 2$ mixed ANOVA with the between-participants factor Learning and the within-participants factors Addend and Block was deployed. Block was included as a factor to explore efficiency patterns between learning and choice block. Our focus was on internal efficiency as dependent variable since, in line with Compton and Logan (1991), we expected learning to increase both speed and accuracy. Inverse efficiency is defined as the reaction time of correct responses divided by the accuracy of all responses (Townsend \& Ashby, 1978) and thus captures both measures of interest simultaneously. To enable exploration of possible speed-accuracy-tradeoffs, we also report speed and accuracy data.

Learning, Addend, and Block interacted in their influence on inverse efficiency $(F(2$, $\left.194)=43.0, p_{G G}=8.04 \times 10^{-13}, \eta_{\mathrm{G}}{ }^{2}=.046\right)$. All other effects were also significant at the .05 significance level and are reported in more detail in Table $\mathbf{S 1}$ in the supplemental materials. 
The three-way interaction reflects the nontrivial consequences of introducing the external resource in the choice block. More specifically, dependent post-hoc t-tests revealed that participants in the Learning 2 condition were, as expected, more efficient in solving equations with Addend 2 in comparison to the control equations with Addend $3(t(50)=10.50, p=3.04$ x $10^{-14}, M_{\text {Delta }}=1120 \mathrm{~ms}$ ) and less efficient in solving equations with Addend 4 in comparison to the control equations with Addend $3\left(t(50)=6.96, p=6.85 \times 10^{-9}, M_{\text {Delta }}=1132 \mathrm{~ms}\right)$; Figure 2a, left. The reverse was true for participants in the Learning 4 condition: they were more efficient in solving equations with Addend 4 in comparison to the control equations with Addend $3\left(t(47)=3.96, p=2.55 \times 10^{-4}, M_{\text {Delta }}=493 \mathrm{~ms}\right)$. However, they were also more efficient in solving equations with Addend 2 in comparison to the control equations with Addend $3\left(t(47)=4.53, p=4.05 \times 10^{-5}, M_{\text {Delta }}=429 \mathrm{~ms}\right)$, which might be due to the high efficiency of the counting strategy for equations with Addend 2 and despite the low efficiency of the retrieval strategy. There was no evidence for inverse efficiency differences for equations with the control Addend 3 but differential Learning conditions (independent t-test: $t(97)=$ $\left..94, p=.351, M_{+2}=3112 \mathrm{~ms}, M_{+4}=2944 \mathrm{~ms}\right)$.

In sum, these results confirm that learning specific equations indeed established efficient internal information access for these equations. The learning-driven rise in efficiency originated from both increased speed and increased accuracy simultaneously; Figure $\mathbf{2 b}$ and c, left. Detailed ANOVA results for inverse efficiency are summarized in the supplemental material, Table S1. ANOVA results for accuracy are also reported in the supplemental materials (Table $\mathbf{S 2}$ and $\mathbf{S 3}$ ) so the reader can further investigate the data for a possible speedaccuracy-tradeoff; there are no signs of a speed-accuracy tradeoff. Note that these results strongly suggest an increase in the retrieval strategy even for the difficult Learning 4 condition, thereby excluding the possibility that the difficult Learning 4 condition draws too many 
processing resources to allow for memory consolidation (as shown for a similar task by Hoyer, Cerella, \& Onyper, 2003).

a

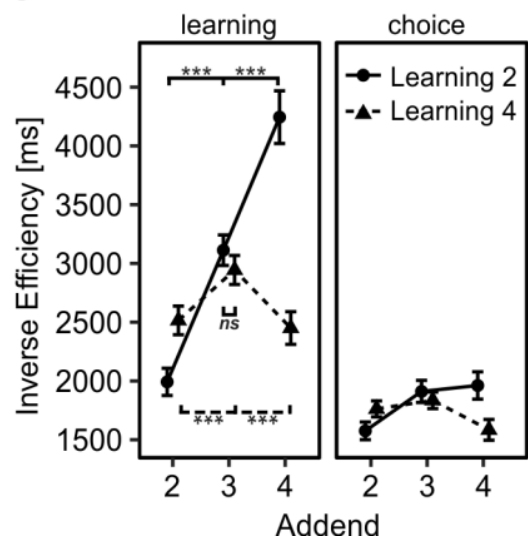

b

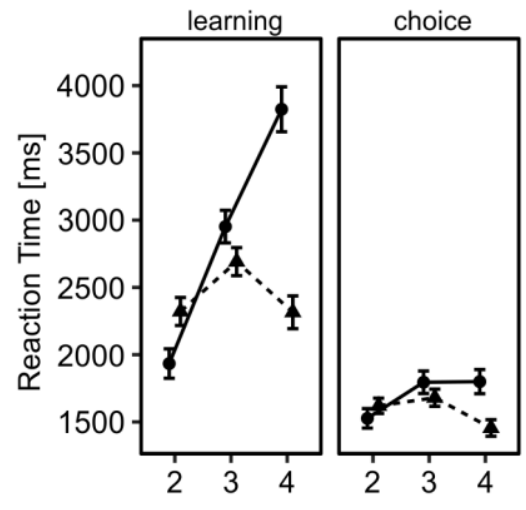

C

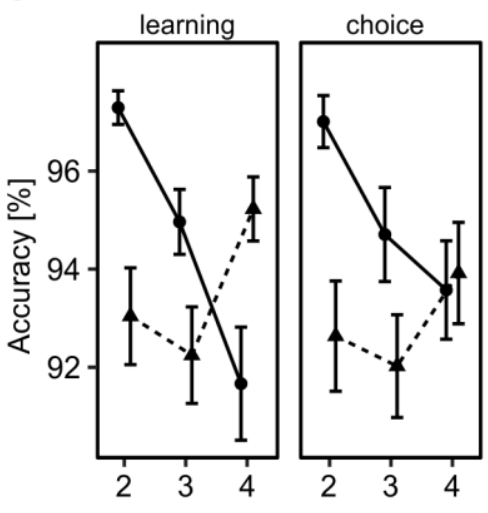

Figure 2. Performance during learning and choice blocks for whole sample. The Learning manipulation successfully altered how efficiently participants could solve a problem internally (a, left). Participants were more efficient in solving the equations they have been learning frequently (Learned 2 and Addend 2; Learned 4 and Addend 4) than in solving control equations (Addend 3). To allow the curious reader to inspect the full data, choice performance (a, right) as well as reaction time (b) and accuracy (c) data is presented as well. Error bars depict SEM. $* * * p<0.001, \mathrm{~ns} p=.35$

Does external retrieval depend on how efficiently solutions can be accessed internally?

External information retrieval is defined as the proportion in which a participant accessed the black box with the mouse cursor during the choice block (Figure 1, right, bottom). As indicated by inverse efficiency during the learning block (Figure 2a, left), participants established efficient internal access to the solutions of frequently but not infrequently practiced alphanumerical problems. Here, we expect the learning effect indicated by the inverse efficiency analysis to be reflected by the frequencies of external information retrieval during the choice block.

To test this hypothesis, a 3 x 2 mixed ANOVA with external information retrieval (in $\%$ of trials) as DV, Addend as within-participants factor, and Learning as between- 
participants factor was used. Only data from the choice block was included in the analysis. The ANOVA was followed up with dependent and independent t-tests where appropriate.

Results of an initial analysis with all participants showed the hypothesized interaction between Learning and Addend $\left(\left(F(2,194)=12.7, p=6.85 \times 10^{-6}, \eta_{\mathrm{G}}{ }^{2}=.005\right)\right.$. However, data exploration revealed that a significant proportion of participants hardly showed any variance in their external information retrieval; Figure 3a. Seventeen participants used the external information in less than 5\% of trials (internal group) of the choice block and thirty-two participants in more than $95 \%$ (external group). Since these two groups exhibit little variance to be explained by the experimental manipulations and introduce normality assumption violations for our analyses, we decided to limit the current analysis to participants that used external information in at least $5 \%$ and at most $95 \%$ of trials in the choice block (mixed group). For the curious reader, performance data for all groups and both blocks is depicted in Figure 3b. Post-hoc t-tests for the mixed group inverse efficiency scores mirror the results obtained from the whole sample. Also note that, at least on a descriptive level, a participant's choice to avoid internal or external strategies was likely at least somewhat adaptive: participants who decided to avoid the internal strategies (i.e., External Group in Figure 3a) performed especially poor in the internal learning block (External Group: learning in Figure 3b).

Results for the mixed group confirm the initial whole sample analysis in showing that Learning and Addend interacted in their influence on external information retrieval $(F(2,96)$ $\left.=12.26, p=1.81 \times 10^{-5}, \eta_{\mathrm{G}}^{2}=.025\right)$. There also was a main effect of Addend $(F(2,96)=$ $\left.\left.8.96, p=4.76 \times 10^{-4}, \eta_{\mathrm{G}}^{2}=.017\right)\right)$ but no main effect of Learning $(F(1,48)=0.07, p=.790$, $\left.\eta_{\mathrm{G}}{ }^{2}=.001\right)$ ). Cell means are illustrated in Figure 4a. A post-hoc dependent t-test confirmed that participants in the Learning 2 condition used external information less when solving equations with the well-learned Addend 2 than with control Addend $3(t(27)=5.07, p=2.54$ 
x $10^{-5}, M_{\text {Delta }}=18.4 \%$ ). Participants in the Learning 4 condition analogously used external information less when solving equations with well-learned Addend 4 than when solving control equations with control Addend $3\left(t(21)=2.82, p=0.0103, M_{\text {Delta }}=10.5 \%\right)$. Thus, participants that established highly efficient internal access to the solution of the respective alphanumerical problem relied less on external information. Surprisingly, there was no difference in external information retrieval between problems with medium and problems with low internal accessibility (Learning 4 and Addend 2 vs Learning 4 and Addend 3: $t(21)=0.80, p=$ $.430, M_{\text {Delta }}=2.7 \%$; Learning 2 and Addend 4 vs Learning 2 and Addend 3: $t(27)=.19, p=$ $\left..849, M_{\text {Delta }}<0.1 \%\right)$. However, when considering that using the counting strategy is faster for Addend 3 than Addend 2 (as reflected by Figure 3c), the missing external retrieval differences for Learning 4 are not that surprising anymore. For Learning 2 however, this explanation does not hold: quite in contrast, the counting strategy is slower for Addend 4 and equations with Addend 4 additionally had been learned less. This will be discussed in more detail in the next section. External information retrieval for the control condition, i.e. trials with the Addend 3, did not differ between Learning conditions $\left(t(48)=0.48, p=0.641, M_{+2}=55.4 \%\right.$, $\left.M_{+4}=51.0 \%\right)$. Thus, there is no evidence for differences in baseline performance between the Learning groups. To account for aberrations from normality, robust non-parametric Wilcoxon signed rank tests and a Wilcoxon rank sum test were used to confirm the results of the significant t-tests.

In sum, these results confirm our hypothesis in that problem solvers adjusted external information retrieval based on how efficiently they can access solutions using internal strategies. However, our data shows one aberration from this pattern. Participants in the Learning 2 condition were significantly less efficient in solving equations with Addend 4 than equations 
with Addend 3 and nevertheless exhibited no differential external information retrieval. This aberration will be addressed in the next section. 
a
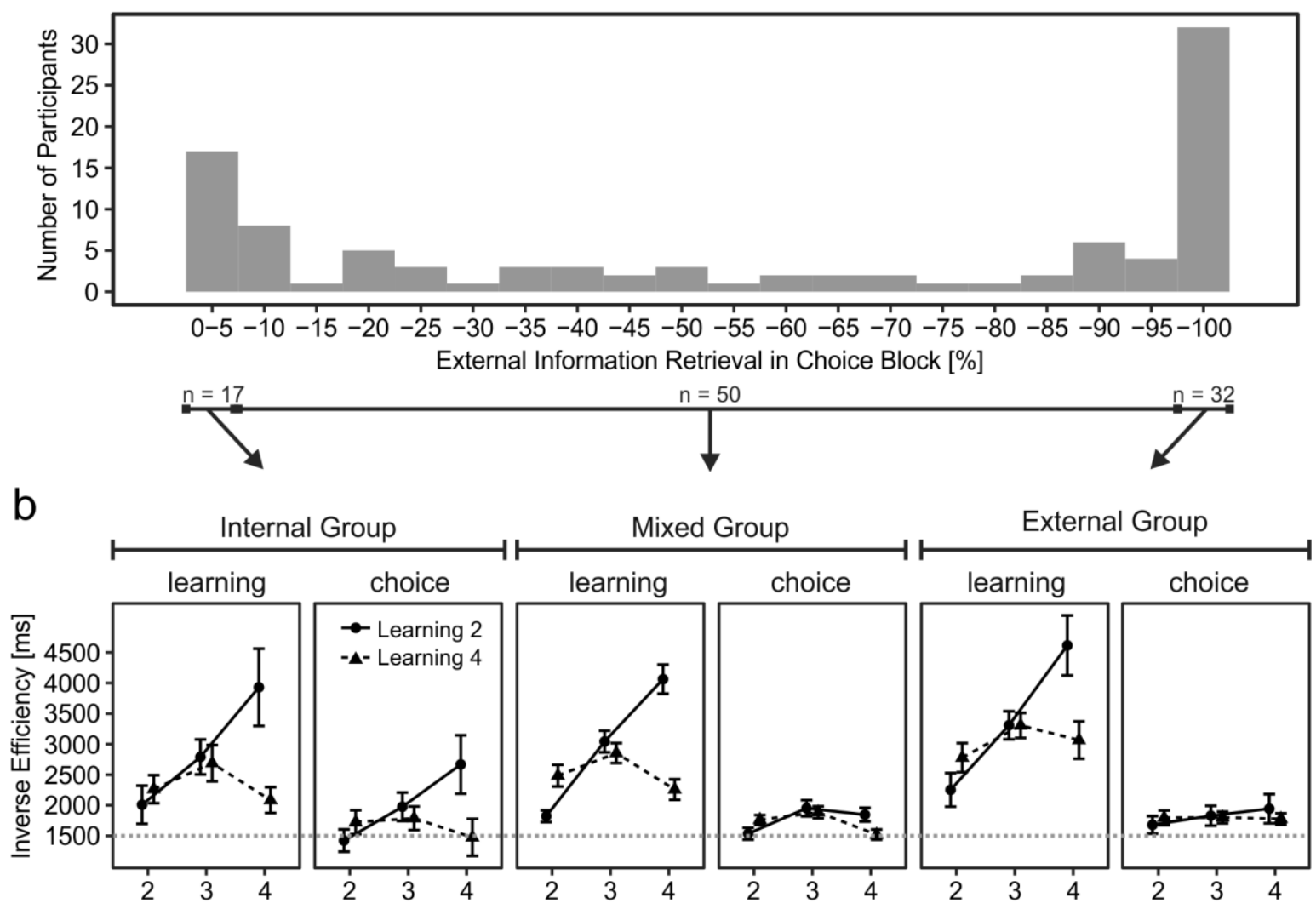

C
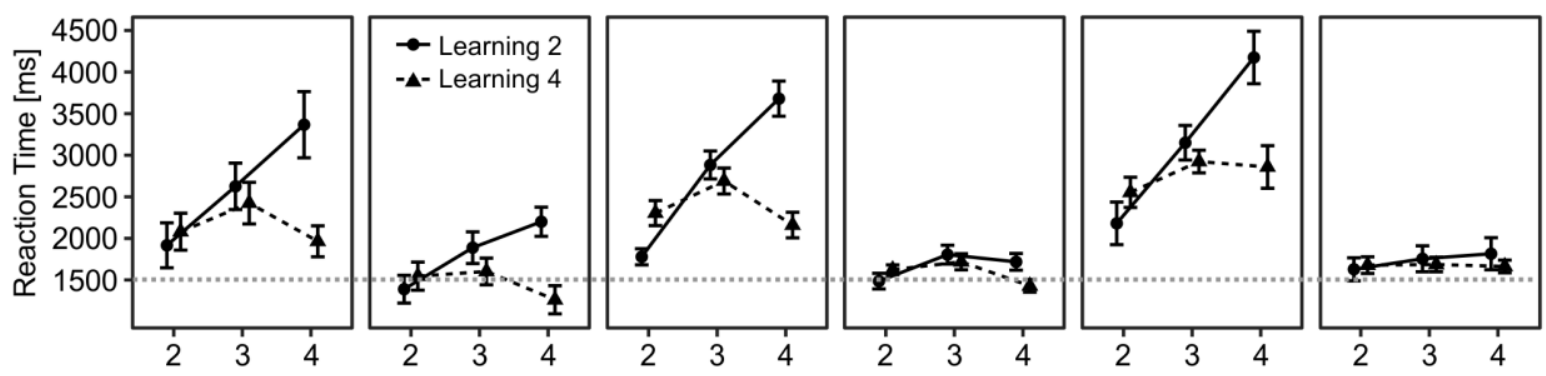

d
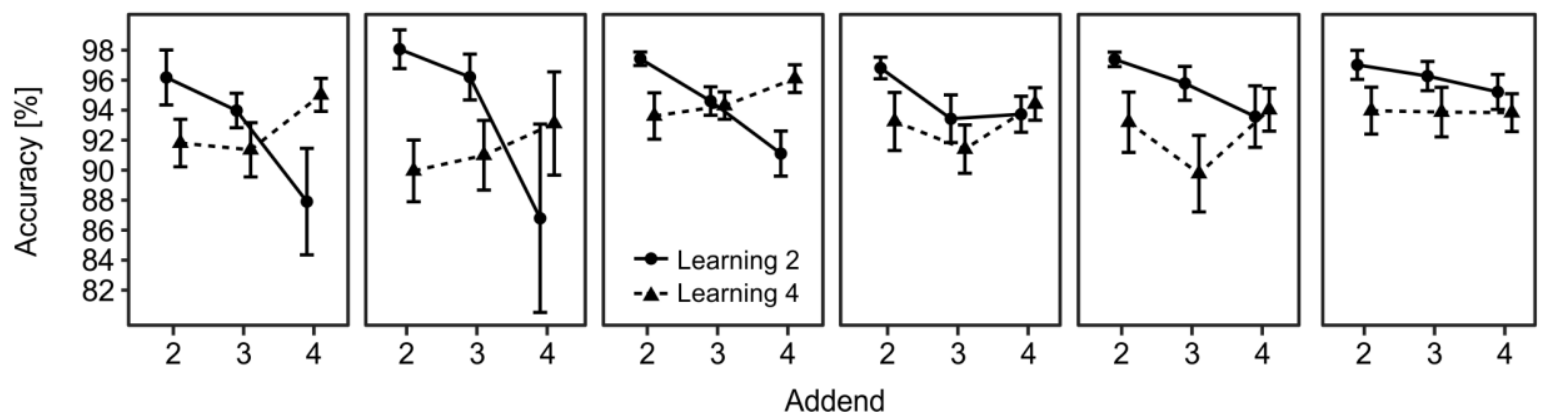

Figure 3. Performance during learning and choice blocks split by groups based on frequency of external information retrieval. Many participants showed hardly any or permanent external information retrieval (a). Performance data was therefore split into three different groups to allow eyeballing of possible performance differences between groups and to allow for a more powerful analysis of external information retrieval data (b, c, d). The figure depends on the same data as Figure 2. Error bars depict SEM. 
a

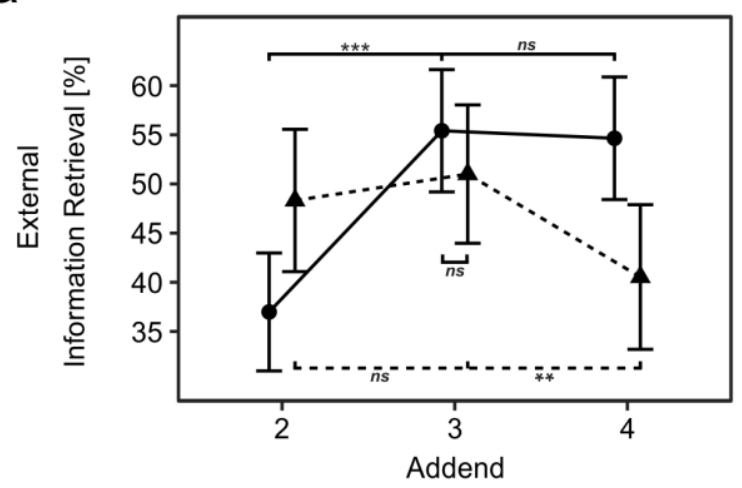

b

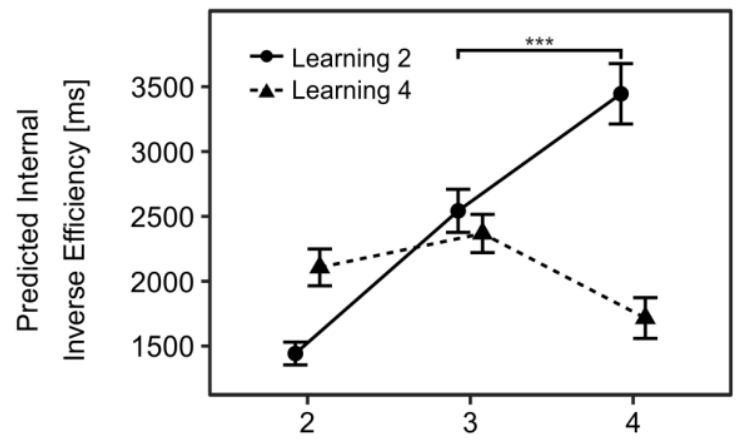

Figure 4. Comparison of external information retrieval and predicted efficiency of internal strategies for the mixed group. Learning and Addend interacted in their influence on external information retrieval (a). The predicted efficiencies for the internal strategies mostly mirror the pattern of external information retrieval (b). It thus appears likely that internal information accessibility informed the decision to retrieve information externally. However, note that participants in the Learning 2 condition exhibit great performance differences between Addend 3 and 4 that are unexpectedly not mirrored in external information retrieval. For the definition of the mixed group, see Figure 3. Error bars depict SEM. Note that the error bars only provide information about effects between, not within, participants. ${ }^{* * *} p<0.001,{ }^{* *} p<=.01, n s \mathrm{p}>.1$

Why does external retrieval not exclusively depend on internal information accessibility?

The following analysis was conducted to explain an unexpected finding: participants in the Learning 2 condition exhibit comparable external information retrieval for Addend 3 and for Addend 4 (Figure 3a) despite prominent differences in inverse efficiencies during the learning block (Figure 2a: learning). However, average inverse efficiencies during the learning block as reported in Figure 2a: learning do not factor possible practice effects throughout the block in. Thus, the poor average internal performance in the "Learning 2, Addend 4" condition reported in Figure 2a: learning might be an artifact stemming from poor initial 
internal performance that had been compensated for by the end of the learning block. To account for this possibility, we fitted the power law function

$$
R T \text { or Accuracy }=a * t^{-k}
$$

to individual accuracy and reaction time data as it developed over time during the learning block. Based on the two fitted functions for each participant, we computed the participant's predicted inverse efficiency for the first trial in the choice block.

Qualitatively, results of a two-factorial mixed ANOVA (Addend x Learning) were comparable with the results obtained by the ANOVA on the inverse efficiency averages, i.e. Addend and Learning interacted in their influence on predicted inverse efficiency $(F(2,96)=$ $51.91, p_{G G}=1.67 \times 10^{-13}, \eta_{\mathrm{G}}^{2}=.270$; see Figure 4b). There also were main effects of Learn$\operatorname{ing}\left(F(1,48)=4.68, p=.0356, \eta_{\mathrm{G}}{ }^{2}=.060\right)$ and Addend $\left(F(2,96)=35.34, p_{G G}=2.10 \times 10^{-10}\right.$, $\left.\eta_{\mathrm{G}}{ }^{2}=.201\right)$. Participants in the "Learning 2, Addend 4" condition were still predicted to perform worse than participants in the "Learning 2, Addend 3" condition $(t(27)=4.55, p=1.01$ x $10^{-4}, M_{\text {Delta }}=903 \mathrm{~ms}$ ), despite the differences found for external information retrieval. Thus, we currently have no data-backed explanation for this unexpected finding but will refer to possible other theoretical considerations in the Discussion.

Exploration: how did participants establish adaptive external information retrieval?

Except for the single unexpected finding reported above, participants reduced their external information retrieval selectively for equations for which high internal information accessibility had been established. To explore possible underlying mechanisms, four exploratory analyses were conducted.

(1) Do participants evaluate addend-specific learning frequencies and adjusted external information retrieval accordingly? In a post-experimental survey, we asked participants whether they preferred internal or external information retrieval and to briefly explain 
why they preferred the one over the other in an open answer format. Interestingly, no single participant mentioned differential learning frequencies for equations with different addends. Participants frequently mentioned that one strategy was "quicker" or "faster" (35 times) or needed less "effort" or was "easier" (26 times). They however did not report any addend-specific strategies. Although we did not explicitly ask participants whether they noticed the Learning manipulation, the fact that no single participant mentioned it during the survey lets it appear unlikely that a strategy based on conscious reflection about the learning process is the prime reason for the adaptive external resource use.

We aimed to confirm this interpretation in a follow-up study. Specifically, we hypothesized that participants did not consciously notice the "learning" manipulation, which would make strategy selection based on a conscious metacognitive evaluation of stimulus frequencies highly unlikely. In the follow-up study, participants followed the identical experimental procedure as in Experiment 1 with one exception: after the learning block, participants now were to judge how frequently they saw equations with different Addends. Specifically, participants had to answer the question "How often have you been solving problems involving ' $+X$ "” three times, where $X$ would be 2,3 , or 4 , respectively. Answers were to be given via a cross on a paper-based visual analogue scale labelled "not at all" on the left-hand and "very often" on the right-hand side.

The sample consisted of eighteen participants that were drawn from the same student population as the main study, were at least 18 years old, reported normal or corrected normal vision, and did not participate in the main experiment. The Ethics Committee at George Mason University approved the experiment and participants provided informed consent prior to participation. Two participants had to be excluded from analysis due to low accuracy in the learning block $(<85 \%$ correct $)$, leading to a final sample size of six- 
teen (nine Learning 2, seven Learning 4; 10 females, mean age: 19.9, age range: 18 - 26, 15 right handed). Only perceived performance will be reported because performance in the learning and choice blocks is not of primary interest for question at hand.

Results of a mixed ANOVA showed that Addend and Learning interacted in their influence on perceived frequency $\left(F(2,28)=12.58, p=1.27 \times 10^{-4}, \eta_{\mathrm{G}}{ }^{2}=.22\right.$; see Figure 5). The main effects did not reach significance at the .05 alpha level (both $p>.59$ ). These results imply that participants were sensitive to changes in learning frequency, which falsifies our initial hypothesis.

Thus, possibly, our participants relied on perceived frequency to decide for a specific cognitive strategy. We now wanted to explore whether perceived frequency can explain the aberration in Experiment 1, i.e. that participants in in the Learning 2 condition did not access the external information more frequently for Addend 4 than for Addend 3. A post-hoc dependent t-test revealed that the perceived frequency data cannot explain the aberration: while participants in the Learning 2 condition of Experiment 1 showed no difference in external information retrieval for Addend 3 and 4, participants in the follow-up study were sensitive to the different learning frequencies (Learning 2 and Addend 3 vs Learning 2 and Addend 4: $\left.t(8)=2.72, p=.0262, M_{\text {Delta }}=15.4 \%\right)$.

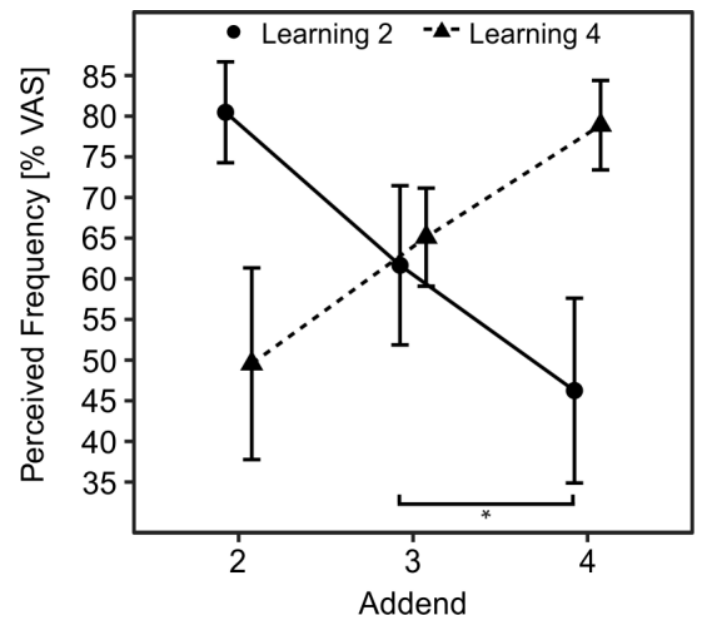


Figure 5. Perceived frequency of alphanumerical equations during the learning block. Note that this graph is based on data from a follow-up study (see text). VAS: visual analogue scale

In sum, this exploration shows that participants did not consciously report an Addend-specific metacognitive strategy to select between internal and external information retrieval. However, data from the follow-up analysis shows that participants are sensitive to the Learning manipulation, which is a prerequisite for any Addend-specific selection strategy. We conclude that the sensitivity to the Learning manipulation might have contributed to strategy selection, though likely not on a conscious level. Instead, strategy selection might have been influenced implicitly by the familiarity of the specific items (Onyper et al., 2006; Schunn et al., 1997).

(2) Do participants rely on performance monitoring to establish adaptive external information retrieval? If participants relied on performance monitoring, adaptive use of external information should emerge only after participants got the chance to compare their performance when using internal with their performance when using external information retrieval. To address this exploratory hypothesis, we looked at the time course of external information retrieval in the choice block. First, to reduce noise in the visual representation, we averaged across four adjacent trials for each Addend separately. Second, we ran two ANOVAs with the within-participants factor Addend on the averages of the first four trials separately for both Learning conditions. For this analysis, only participants in the mixed group were used. We did not run one combined ANOVA instead since we were not interested in a possible main effect of Learning or the interaction between Learning and Addend. 
Results indicate that Addend significantly influenced external information retrieval during the first four choice trials for both Learning $2\left(F(2,54)=10.13, p=1.84 \times 10^{-4}\right.$, $\left.\eta_{\mathrm{G}}{ }^{2}=.108\right)$ and Learning $4\left(F(2,42)=6.74, p=.00290, \eta_{\mathrm{G}}{ }^{2}=.104\right)$. Post-hoc dependent t-tests confirmed that participants in the Learning 2 condition used the external information less with respect to the control condition (i.e., Addend 3) when solving equations for whose solutions high internal accessibility had been established $(t(27)=3.34, p=$ $0.00248, M_{+3}-M_{+2}=21.4 \%$ ); Figure 6a. The same difference was trending for participants in the Learning 4 condition $\left.\left(t(21)=2.02, p=0.0563, M_{+3}-M_{+4}=12.1 \%\right)\right)$ group; Figure 6b. Given that decreased external information retrieval for well-learned solutions was exhibited already at the very beginning of the choice block, these results let it appear unlikely that the adaptive behavior was based on performance monitoring during the choice block. ${ }^{6}$

a

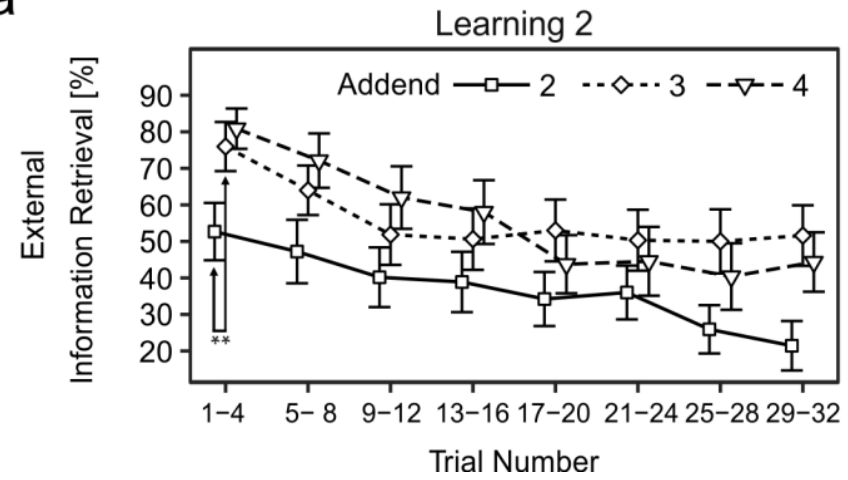

b

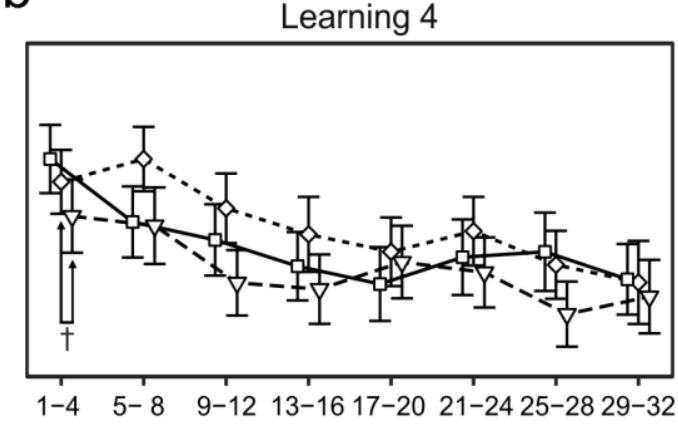

Figure 6. Time course of external information retrieval during choice block for the Learning 2 (a) and the Learning 4 (b) condition. Error bars depict SEM. $* * p<.01, \uparrow p=.06$

\footnotetext{
${ }^{6}$ Also note that the negative slope exhibited in Figure 6 suggests an ongoing increase in efficiency of internal information retrieval. To allow the interested reader to inspect the time course of performance during the choice block, we also provide graphs illustrating inverse efficiency, reaction time, as well as accuracy in the supplemental materials (Figures S1, S2, and S3, respectively; note the roughly constant internal performance over time despite the decreasing use of external information retrieval).
} 
(3) Do participants rely on parallel strategy use to establish the adaptive external information retrieval? Participants might have executed internal and external strategies at the same time and have used the solution of whichever strategy was finished first (i.e., a race between strategies Compton \& Logan, 1991; Logan, 1988). If so, our finding of decreased external information retrieval for equations with high internal accessibility might be due to the fact that internal solution retrieval was faster than accessing the solution externally (i.e., faster than moving the cursor from the starting position to the black box). To investigate this post-hoc hypothesis, we compared (a) the count of trials in which participants did not move the cursor for even a single pixel to (b) the count of trials in which participants started to move the cursor but did not reach the black box and to (c) the count of trials in which the cursor was moved inside the black box.

Out of all trials across all participants, a substantial proportion acquired the solution internally without even initiating the external strategy (a: 3168 trials or $33.7 \%$ ). Thus, in a third of trials, participants did unambiguously not rely on parallel processing. This upfront preference for internal strategies might be due to an initial strategy selection phase that is informed by higher-level metacognitive evaluations (e.g., being convinced of one's own abilities, Gilbert, 2015; or being suspicious about the usefulness of the external strategy, Weis \& Wiese, 2019) or lower-level item-specific or strategy-specific learning (as claimed by the CMPL, Rickard, 1997; and the ASCM, Siegler \& Lemaire, 1997 models). If we are willing to assume that participants have not started moving the mouse without cognitive intent (e.g., due to muscle jitter), results however also show that participants did sometimes use both strategies in parallel (b: 615 trials or $6.5 \%$ ). This result is in line with a study by Walsh and Anderson (2009): arithmetic problem solvers sometimes started moving the mouse towards a screen-based calculator but changed their trajectory towards 
the answer box before reaching the calculator. Lastly, as already indicated by the main analysis, the external strategy was fully executed for most trials (c: 5619 trials or 59.8\%). However, with the current data, it is impossible to tell whether participants followed a purely external or a parallel strategy during these trials. Nevertheless, the existence of parallel strategy execution (b; Walsh \& Anderson, 2009) and the fact that some participants mentioned that they used the external strategy specifically to save effort (see Exploration: Do participants evaluate individual learning frequencies metacognitively and adjusted external information retrieval accordingly) makes it likely that participants made use of both purely external and parallel options, though the exact proportions cannot be determined with the current data. The present analysis thus suggests the existence of all possible, i.e. purely internal, purely external, and parallel, processing strategies.

(4) Did participants mostly rely on a sequential strategy to establish the adaptive external information retrieval? Participants might have tried to recall the solution first internally and only in a second step consider other options. Such a sequential strategy has been suggested to be the "best of both worlds": it does improve retrieval from internal memory even when the recall fails and still makes use of the external strategy to omit costly internal strategies like counting (Pyke \& LeFevre, 2011). Here, we investigate this exploratory hypothesis using mouse movement onset data. The general idea is that if participants showed different mouse movement onsets depending on their internal information accessibility (compare Figure 4b) it would speak for some sequential mechanism. Else, there would be no reason for onsets to be different. We now present one possible underlying mechanism for differential mouse movement onsets:

The higher the internal information accessibility, the faster and more accurate internal retrieval becomes (e.g., Logan, 1988; Ratcliff, 1978). However, analogously, the higher 
internal information accessibility is, the earlier a problem solver might stop the retrieval process and continue with another (in the present case, internal counting or external mouse movement) strategy. In other words, if one "knows" that internal accessibility should be high for a specific solution, one would predict an earlier retrieval success and might be willing to declare the retrieval as unsuccessful earlier. Such a prediction would also be made by a 2-choice diffusion model with the choices 'retrieval' and 'retrieval error': error RT with a high drift parameter (as for frequently learned alphanumeric equations) would be predicted to be lower than error RT with a low drift parameter (as for infrequently learned alphanumeric equations; Ratcliff \& McKoon, 2008). Note that this sort of decision process would imply that participants learned to associate specific features (e.g. " $+4 ”)$ with specific drift rates. Such an assumption is not implausible as problem solvers are known to be sensitive to "featural data" (Siegler \& Lemaire, 1997, p. 72) and to use such data to inform their cognitive strategy selection for novel problems (as claimed by the ASCM, e.g. Siegler \& Lemaire, 1997). Following that rationale, we hypothesize that if participants relied on a sequential strategy, they should have started moving the mouse earlier for well-trained equations than for less trained equations.

A mixed ANOVA (Addend x Learning) and one-sided post-hoc dependent t-tests were used to test this explorative hypothesis. Trials with mouse movement onsets deviating more than three standard deviations from the individual mean were filtered. Only participants in the mixed group were used for this analysis. Six participants were excluded because they moved the mouse less than three times in at least one of the three Addend conditions. We chose a threshold of three trials as a liberal criterion to avoid noisy individual estimates. 
In accordance with the hypothesis, Addend and Learning interacted in their influence on mouse movement start $\left(F(2,84)=3.37, p=.0390, \eta_{\mathrm{G}}{ }^{2}=.018\right)$; Figure 7a. Posthoc tests confirmed that participants in the Learning 2 condition started moving the mouse earlier for equations with Addend 2 than with Addend $4(t(25)=2.37, p=0.0128$, $\left.\left.M_{+4}-M_{+2}=89 \mathrm{~ms}\right)\right)$. The reverse comparison was trending for participants in the Learning 4 condition $\left.\left(t(17)=1.47, p=0.0805, M_{+2}-M_{+4}=55 \mathrm{~ms}\right)\right)$. These results suggest that our problem solvers, at least in some trials, used a sequential approach in which they first tried to recall the solution from internal memory and only then started considering other options. Given the exploratory nature of this analysis, this result should not be overinterpreted.

To explore possible differences between groups (see Figure 3) we also include an analogous ANOVA for the external group. Results showed no interaction effect $(F(2,60)$ $\left.=.41, p_{\mathrm{GG}}=.596, \eta_{\mathrm{G}}{ }^{2}<.001\right)$; Figure 7b. The main effect of Learning was trending $(F(1$, $\left.30)=3.69, p=.0642, \eta_{\mathrm{G}}{ }^{2}=.11\right)$ and there was no main effect of Addend $(F(2,60)=.29$, $\left.p_{\mathrm{GG}}=.672, \eta_{\mathrm{G}}^{2}<.001\right)$. The results for the external group suggest that these participants did not rely on a sequential strategy when deciding which strategy to use. Results rather suggest that participants decided for the external strategy early on and further on did not try to rely on internal retrieval at all.

a

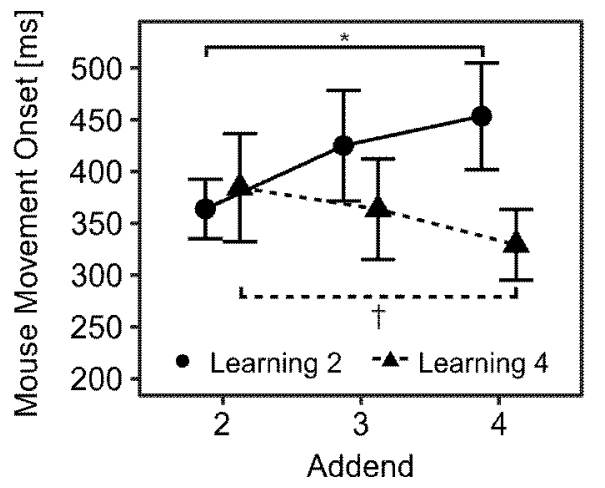

$\mathrm{b}$

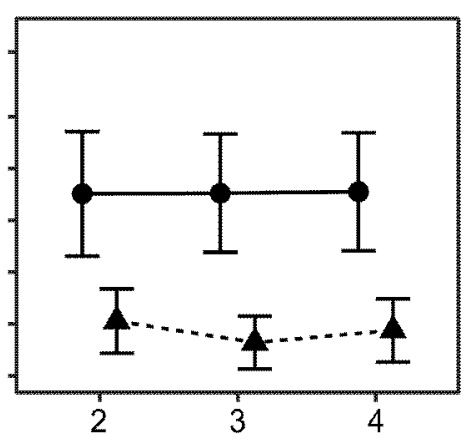


Figure 7. Mouse movement onset in the choice block. Data is shown separately for the mixed (a) and the external (b) group. Error bars depict SEM. * $p=.01, \dagger p=.08$

\section{Discussion}

In the present study, a novel human-computer-interaction paradigm was used to investigate whether problem solvers choose between internal and external information retrieval based on the accessibility of internal information. By and large, we found this to be the case: increasing the internal accessibility of a problem's solution decreased how frequently participants retrieved information externally. Crucially, this relationship was present even when visual features of a task would suggest the opposite (e.g., "A + 4" looks harder to solve internally than " $\mathrm{A}+2$ " but is nevertheless solved more frequently internally when the solution's internal accessibility is high). Participants thus were sensitive to their internal information access and used that sensitivity to choose between internal and external cognitive strategies rather than using sensitivity-independent strategies based on the task's visual features. Four exploratory analyses were conducted that (1) suggest adaptive choice between internal and external information retrieval to be, at least in some instances, realized using a sequential "try internal retrieval first and then consider other options" heuristic; (2) are inconclusive about whether the decision between internal and external retrieval was additionally dependent on an implicit metacognitive process that evaluates differential learning frequencies or familiarity in the learning block, (3) let it appear unlikely that the choice was dependent on performance monitoring during the choice block, and (4) suggest that internal and external retrieval are not executed in parallel in some but might be in other instances. 
Results are consistent with previous studies suggesting humans to be impartial about whether to use internal or external strategies for cognitive processing (Gray \& Fu, 2004; Gray et al., 2006; Morgan et al., 2009), ultimately preferring the strategy with the lower costs. Results extend their findings by providing possible underlying mechanisms of strategy choice. Results also support theories that proclaim the cognitive system to be rational and adaptive (e.g., Anderson, 1990) and question theories that proclaim a strong bias against mental effort (e.g. Ballard, Hayhoe, Pook, \& Rao, 1997; Kool, McGuire, Rosen, \& Botvinick, 2010). How do problem solvers decide between internal and external cognitive strategies? Previous studies suggest that problem solvers might rely on knowledge about the efficiencies of differential internal strategies that might be partially based on the analysis of the problem's visual features (Bourne et al., 2010; Lemaire \& Reder, 1999; Siegler \& Lemaire, 1997), on the familiarity of the specific problem (Schunn et al., 1997), or subjective judgments of own ability (Gilbert, 2015; Touron, 2015). Problem solvers might also rely on performance monitoring using error feedback and/or response time (for a review, see Ullsperger, Fischer, Nigbur, \& Endrass, 2014) or follow less monitoring-sensitive strategies like trying to access the solution via internal and external strategies in parallel. Conversely, problem solvers might first try to recall the information from memory and, if that fails, access the solution via the external resource in a second step (for competing internal strategies, the parallel option seems more plausible; Logan, 1988). The current study allows us to compare the strategy choice process for the alphanumeric task at hand with what has been proposed in these previous studies:

(1) Given that no addend-specific strategies were reported in the questionnaire at the end of the study, we find it to be unlikely that our participants made their adaptive decision to use an external strategy based on a conscious metacognitive strat- 
egy. However, additional data showed that participants were sensitive to the addend-specific Learning manipulation (i.e., knew which types of equations they learned more and which ones they learned less frequently), which would enable them to base their strategy choice on the familiarity of the problem (i.e., use the external strategy for unfamiliar problems; Schunn et al., 1997). Thus, at this point, it is unclear whether our participants have implicitly used that sensitivity to inform strategy selection.

(2) We deem it unlikely that performance monitoring during the choice block was the prime cause for adaptive external strategy use given that the frequency of external information retrieval was adjusted to the Learning condition right from the beginning of the choice block.

(3) It appears equally unlikely that participants consistently executed internal and external retrieval in parallel since in about a third of all trials the mouse was not moved at all. This clear preference for the internal strategy in about a third of the trials is consistent with findings of a study that used mouse trajectories to examine problem solvers' uncertainty about whether to use an internal or an external strategy: participants had to solve math equations and could either move their mouse towards a calculator first (i.e., external strategy) or immediately towards the answer box (i.e., internal strategy). Though participants sometimes adjusted their mouse movement throughout the trial, they also frequently and unambiguously preferred not to use the external resource at all, as indicated by a mouse trajectory directly leading towards the answer box without any curvature towards the calculator (Walsh \& Anderson, 2009). 
(4) We investigated whether our problem solvers might have used a sequential mechanism where participants retrieve the solution externally only if internal retrieval had failed beforehand. This sequential mechanism was supported by our data: in accordance with theoretical predictions based on a diffusion model with the outcomes "retrieval" or "retrieval error" (see Results: Exploration (4) Did participants mostly rely on a sequential strategy to establish the adaptive external information retrieval?), our problem solvers started external retrieval (i.e., mouse movements) earlier for frequently than for infrequently trained equations. A similar sequential mechanism has been reported for strategy choice in mental arithmetic: people likely first tried to verify equations using the "five rule" before engaging in standard arithmetic operations (Lemaire \& Reder, 1999). Interestingly, this effect vanished for participants that almost exclusively relied on external retrieval, thus suggesting a different mechanism for those participants.

Taken together, the exploratory findings suggest a divergence of how internal (e.g., memory retrieval and mental arithmetic) and competing mixed (e.g., memory retrieval and external retrieval) strategies are employed. Internal strategies might be more prone to be employed in parallel (Logan, 1988; but also see Lemaire \& Reder, 1999, for sequential processing) while the existence of even comparably easy external strategies like the one used in the current paradigm might encourage solitarily strategy use (current study). Future studies are needed to consolidate this finding and should also address the underlying reasons, for example the possibility that external strategies are oftentimes too resource-draining to allow simultaneous execution of internal strategies. 
Though we deem it likely that a sequential mechanism was frequently used for strategy selection in the current study, we want to stress that we do by no mean deny the existence of other mechanisms, for example:

(1) Monitoring accuracy and time feedback during the learning block. However, note that speed- and accuracy-related performance does not exhaustingly predict strategy selection (Gray et al., 2006; Risko et al., 2014; Walsh \& Anderson, 2009; Weis \& Wiese, 2019), which was also true in the present study: even though participants in the Learning 2 condition relied less on the external strategy when internal accessibility of the solution was high (i.e., Addend 2) rather than medium (i.e., Addend 3), this behavior was not mirrored when comparing medium with low (i.e., Addend 4) accessibilities; Figure 4a.

(2) Metacognitive misconceptions about their performance. The behavior might not have been mirrored because of people's metacognitive misconceptions about their performance (Dunn \& Risko, 2016; Pauszek \& Gibson, 2018; Risko \& Dunn, 2015; Weis \& Wiese, 2019): our participants might have underestimated how slow they are at solving equations which they had little experience with.

Further research is needed to clarify the interplay of different parameters like monitoring-based efficiency optimization and metacognitive misjudgments on external strategy use. Equally importantly, it is yet to be examined whether findings in the domain of declarative long-term memory, like in the current study, transfer to other areas of cognition like working memory or spatial navigation (see also Risko \& Gilbert, 2016, p. 685) and if similar efficiency-dependent mechanisms of external strategy use hold when outsourcing memory to humans (i.e., transactive memory; Wegner, 1987) rather than computers. Lastly, we want to direct the reader's attention towards the fact that about half of our participants (i.e., the inter- 
nal and external group) exhibited hardly any variance in strategy choice, which would be compatible with the view that many participants make a strategy choice once rather than at the beginning of each trial (similar to some participants in Bourne et al., 2010). Understanding the individual differences in cognitive strategy choice will be key to an improved understanding of how humans solve problems in cognitive environments (as discussed by Risko \& Gilbert, 2016).

From an applied perspective, the present results inform possible intervention methods aimed at remediating external information retrieval in particular and, possibly, external strategy use in general. Currently, such intervention methods are hardly available (Risko \& Gilbert, 2016, p. 685). From the current data, we can extrapolate that improving the efficiency of internal strategies should by itself suffice to remediate externalization behavior. A similar approach but altering the efficiency of external rather than internal strategies has been suggested by O'Hara and Payne (1998): increasing the time costs associated with using (an interface that was needed for) an external strategy encouraged more frequent internal strategy use. This mechanism of decreasing efficiency of an external or increasing efficiency of an internal strategy could be used to guide externalization behavior whenever internal have more favorable attributes than external strategies. For example, bolstering internal strategy use can be important and beneficial when external strategies are regularly unavailable or when insightful knowledge transfer is needed, the latter of which can oftentimes only be achieved internally. A proof of concept for this mechanism, but targeting the efficiency of external rather than internal strategies, was provided by O'Hara and Payne (1998). Analogously, internal strategies should intentionally not be relied upon when they have unfavorable properties. For example, in internal memory, similar stimuli are often grouped together to reduce representational complexity (Nosofsky, 1992), leading to a decreased ability to discriminate attributes 
associated with these similar stimuli and decreased performance in discrimination-based tasks (i.e., similar stimuli are basically underrepresented since they are conceived as one rather than two separate entities). One possibility to restore performance is to rely on external strategies without biased representations (Fu, 2011). Providing easy access to the external strategy (Fu, 2011) and avoiding learning-induced increases in the efficiency of the internal strategy (current study) would guide the user towards the beneficial reliance on external strategies. We thus argue that the efficiency of both internal and external strategies can be intentionally manipulated as to maximize specific performance outcomes in cognitive tasks.

The present findings also have implications for problem solvers in static cognitive environments where properties like task difficulty or external information accessibility cannot be changed. For example, the speed of accessing information online depends heavily on the quality of the internet connection and cannot be directly controlled by the problem solver. In contrast, changing internal information accessibility oftentimes depends on deploying appropriate mental strategies that can be taught or discovered (Lemaire \& Lecacheur, 2001) as well as on learning (e.g., Logan, 1988; or the present study). Both options leave the initiative with the problem solver rather than some extrinsic force like network coverage. The present finding that human problem solvers adjust their use of externally stored information based on the internal accessibility of that information speaks for the human ability to proficiently exploit a technologized environment for their own benefit. Establishing high internal information accessibility is thus a viable option to become less dependent on that environment whenever desired. 


\section{Conclusion}

In an increasingly computerized future, being an efficient problem solver in interactive environments will gain importance. Former studies have shown that increasing the time needed to access externally stored information increases reliance on brain-based information. Here, we strengthen the prevalent notion that increasing the efficiency of brain-based information retrieval increases reliance on brain-based information in an analogous manner and supply possible underlying mechanisms. Our study thereby increases the understanding of human behavior in interactive settings that afford external information storage. 


\section{References}

Anderson, J. R. (1987). Skill acquisition: Compilation of weak-method problem situations. Psychological Review, 94(2), 192.

Anderson, J. R. (1990). The Adaptive Character of Thought. https://doi.org/10.4324/9780203771730

Ballard, D. H., Hayhoe, M. M., Pook, P. K., \& Rao, R. P. (1997). Deictic codes for the embodiment of cognition. Behavioral and Brain Sciences, 20(4), 723-742.

Bourne, L. E., Raymond, W. D., \& Healy, A. F. (2010). Strategy selection and use during classification skill acquisition. Journal of Experimental Psychology: Learning, Memory, and Cognition, 36(2), 500-514. https://doi.org/10.1037/a0018599

Brainard, D. H. (1997). The psychophysics toolbox. Spatial Vision, 10, 433-436. https://doi.org/10.1163/156856897x00357

Clark, A. (1999). An embodied cognitive science? Trends in Cognitive Sciences, 3(9), 345351.

Compton, B. J., \& Logan, G. D. (1991). The transition from algorithm to retrieval in memory-based theories of automaticity. Memory \& Cognition, 19(2), 151-158. https://doi.org/10.3758/BF03197111

Dixon, R. A., \& de Frias, C. M. (2004). The Victoria Longitudinal Study: From Characterizing Cognitive Aging to Illustrating Changes in Memory Compensation. Aging, Neuropsychology, and Cognition, 11(2-3), 346-376.

https://doi.org/10.1080/13825580490511161

Dunn, T. L., \& Risko, E. F. (2016). Toward a Metacognitive Account of Cognitive Offloading. Cognitive Science, 40(5), 1080-1127. https://doi.org/10.1111/cogs.12273 
Faul, F., Erdfelder, E., Lang, A.-G., \& Buchner, A. (2007). G* Power 3: A flexible statistical power analysis program for the social, behavioral, and biomedical sciences. Behavior Research Methods, 39(2), 175-191.

Fu, W.-T. (2011). A Dynamic Context Model of Interactive Behavior: Cognitive Science. Cognitive Science, 35(5), 874-904. https://doi.org/10.1111/j.1551-6709.2011.01173.x

Gilbert, S. J. (2015). Strategic use of reminders: Influence of both domain-general and taskspecific metacognitive confidence, independent of objective memory ability. Consciousness and Cognition, 33, 245-260. https://doi.org/10.1016/j.concog.2015.01.006

Gray, W. D., \& Fu, W.-T. (2004). Soft constraints in interactive behavior: The case of ignoring perfect knowledge in-the-world for imperfect knowledge in-the-head. Cognitive Science, 28(3), 359-382. https://doi.org/10.1016/j.cogsci.2003.12.001

Gray, W. D., Sims, C. R., Fu, W.-T., \& Schoelles, M. J. (2006). The soft constraints hypothesis: A rational analysis approach to resource allocation for interactive behavior. Psychological Review, 113(3), 461-482. https://doi.org/10.1037/0033-295X.113.3.461

Hines, J., Hertzog, C., \& Touron, D. (2012). A prelearning manipulation falsifies a pure associational deficit account of retrieval shift during skill acquisition. Aging, Neuropsychology, and Cognition, 19(4), 449-478.

https://doi.org/10.1080/13825585.2011.630718

Hollan, J., Hutchins, E., \& Kirsh, D. (2000). Distributed cognition: Toward a new foundation for human-computer interaction research. ACM Transactions on Computer-Human Interaction (TOCHI), 7(2), 174-196.

Howes, A., Duggan, G. B., Kalidindi, K., Tseng, Y.-C., \& Lewis, R. L. (2016). Predicting Short-Term Remembering as Boundedly Optimal Strategy Choice. Cognitive Science, 40(5), 1192-1223. 
Hoyer, W. J., Cerella, J., \& Onyper, S. V. (2003). Item learning in cognitive skill training: Effects of item difficulty. Memory \& Cognition, 31(8), 1260-1270. https://doi.org/10.3758/BF03195809

Kool, W., McGuire, J. T., Rosen, Z. B., \& Botvinick, M. M. (2010). Decision making and the avoidance of cognitive demand. Journal of Experimental Psychology: General, 139(4), 665-682. https://doi.org/10.1037/a0020198

Lemaire, P., \& Lecacheur, M. (2001). Older and younger adults' strategy use and execution in currency conversion tasks: Insights from French franc to euro and euro to French franc conversions. Journal of Experimental Psychology: Applied, 7(3), 195-206. https://doi.org/10.1037//1076-898X.7.3.195

Lemaire, P., \& Reder, L. (1999). What affects strategy selection in arithmetic? The example of parity and five effects on product verification. Memory \& Cognition, 27(2), 364382. https://doi.org/10.3758/BF03211420

Logan, G. D. (1988). Toward an instance theory of automatization. Psychological Review, 95(4), 492.

Logan, G. D., \& Klapp, S. T. (1991). Automatizing Alphabet Arithmetic: I. Is Extended Practice Necessary to Produce Automaticity? Journal of Experimental Psychology: Learning, Memory, and Cognition, 17(2), 179-195.

Morgan, P. L., Patrick, J., Waldron, S. M., King, S. L., \& Patrick, T. (2009). Improving memory after interruption: Exploiting soft constraints and manipulating information access cost. Journal of Experimental Psychology: Applied, 15(4), 291-306. https://doi.org/10.1037/a0018008

Neth, H., \& Payne, S. J. (2001). Addition as interactive problem solving. Proceedings of the Annual Meeting of the Cognitive Science Society, 23. 
Norman, D. A. (1988). The psychology of everyday things.(The design of everyday things). Basic Books.

Nosofsky, R. M. (1992). Exemplar-based approach to relating categorization, identification, and recognition. In F. G. Ashby (Ed.), Scientific psychology series. Multidimensional models of perception and cognition (pp. 363-393). Hillsdale, NJ, US: Lawrence Erlbaum Associates, Inc.

O’Hara, K. P., \& Payne, S. J. (1998). The Effects of Operator Implementation Cost on Planfulness of Problem Solving and Learning. Cognitive Psychology, 35(1), 34-70. https://doi.org/10.1006/cogp.1997.0676

Onyper, S. V., Hoyer, W. J., \& Cerella, J. (2006). Determinants of retrieval solutions during cognitive skill training: Source confusions. Memory \& Cognition, 34(3), 538-549.

Pauszek, J. R., \& Gibson, B. S. (2018). The Least Costs Hypothesis: A rational analysis approach to the voluntary symbolic control of attention. Journal of Experimental Psychology: Human Perception and Performance, 44(8), 1199.

Pelli, D. G. (1997). The VideoToolbox software for visual psychophysics: Transforming numbers into movies. Spatial Vision, 10(4), 437-442. https://doi.org/10.1163/156856897X00366

Pyke, A. A., \& LeFevre, J.-A. (2011). Calculator use need not undermine direct-access ability: The roles of retrieval, calculation, and calculator use in the acquisition of arithmetic facts. Journal of Educational Psychology, 103(3), 607-616. https://doi.org/10.1037/a0023291

Ratcliff, R. (1978). A Theory of Memory Retrieval. Psychological Review, 85(2), 59-108. https://doi.org/10.1037/0033-295X.85.2.59 
Ratcliff, R., \& McKoon, G. (2008). The Diffusion Decision Model: Theory and Data for Two-Choice Decision Tasks. Neural Computation, 20(4), 873-922. https://doi.org/10.1162/neco.2008.12-06-420

Rickard, T. C. (1997). Bending the power law: A CMPL theory of strategy shifts and the automatization of cognitive skills. Journal of Experimental Psychology: General, $126(3), 288$.

Risko, E. F., \& Dunn, T. L. (2015). Storing information in-the-world: Metacognition and cognitive offloading in a short-term memory task. Consciousness and Cognition, 36, $61-74$.

Risko, E. F., \& Gilbert, S. J. (2016). Cognitive Offloading. Trends in Cognitive Sciences, 20(9), 676-688. https://doi.org/10.1016/j.tics.2016.07.002

Risko, E. F., Medimorec, S., Chisholm, J., \& Kingstone, A. (2014). Rotating with rotated text: A natural behavior approach to investigating cognitive offloading. Cognitive Science, 38(3), 537-564.

Robbins, P., \& Aydede, M. (2009). A short primer on situated cognition. The Cambridge Handbook of Situated Cognition, 3-10.

Schunn, C. D., Reder, L. M., \& Nhouyvanisvong, A. (1997). To Calculate or Not to Calculate: A Source Activation Confusion Model of Problem Familiarity’s Role in Strategy Selection. Journal of Experimental Psychology: Learning, Memory, and Cognition, 23(1), 3-29. https://doi.org/10.1037/0278-7393.23.1.3

Siegler, R. S., \& Lemaire, P. (1997). Older and younger adults' strategy choices in multiplication: Testing predictions of ASCM using the choice/no-choice method. Journal of Experimental Psychology: General, 126(1), 71. 
Storm, B. C., Stone, S. M., \& Benjamin, A. S. (2017). Using the Internet to access information inflates future use of the Internet to access other information. Memory, 25(6), $717-723$.

Touron, D. R. (2015). Memory avoidance by older adults: When "old dogs" won't perform their "new tricks." Current Directions in Psychological Science, 24(3), 170-176.

Touron, D. R., \& Hertzog, C. (2004). Distinguishing Age Differences in Knowledge, Strategy Use, and Confidence During Strategic Skill Acquisition. Psychology and Aging, 19(3), 452-466. https://doi.org/10.1037/0882-7974.19.3.452

Townsend, J. T., \& Ashby, F. G. (1978). Methods of modeling capacity in simple processing systems. Cognitive Theory, 3, 200-239.

Ullsperger, M., Fischer, A. G., Nigbur, R., \& Endrass, T. (2014). Neural mechanisms and temporal dynamics of performance monitoring. Trends in Cognitive Sciences, 18(5), 259-267. https://doi.org/10.1016/j.tics.2014.02.009

Walsh, M. M., \& Anderson, J. R. (2009). The strategic nature of changing your mind. Cognitive Psychology, 58(3), 416-440.

Wegner, D. M. (1987). Transactive memory: A contemporary analysis of the group mind. In Theories of group behavior (pp. 185-208). Springer.

Weis, P. P., \& Wiese, E. (2019). Using Tools to Help Us Think: Actual But also Believed Reliability Modulates Cognitive Offloading. Human Factors, 61(2), 243-254. https://doi.org/10.1177/0018720818797553

Wilson, M. (2002). Six views of embodied cognition. Psychonomic Bulletin \& Review, 9(4), $625-636$.

Zbrodoff, N. J. (1995). Why is 9+ 7 harder than 2+ 3? Strength and interference as explanations of the problem-size effect. Memory \& Cognition, 23(6), 689-700. 